\title{
ESTUDO DE CAUSA BÁSICA DE OBITOS DE MENORES DE 15 ANOS, OCORRIDOS EM HOSPITAL DE FLORIANÓPOLIS, SC \\ (BRASIL), EM 1982
}

\author{
Geni Machado Strozzi* \\ João Bosco Strozzi** \\ Maria de Lourdes de Souza*** \\ Mauro Duarte Schutel****
}

STrozzI, G.M. et al. Estudo de causa básica de óbitos de menores de 15 anos, ocorridos em hospital de Florianópolis, SC (Brasil), em 1982 Rev. Saúde públ,, S. Paulo, 19 : 123-32, 1985.

RESUMO: Foram analisados atestados de óbito segundo a causa básica. Da totalidade dos óbitos ocorridos em hospital infantil de Florianópolis, SC (Brasil), durante o ano de 1982, 161 foram considerados válidos para o estudo. Através das informações encontradas nos prontuários, os atestados de óbito foram refeitos e, posteriormente, comparados com os originais. Observou-se uma concordância de $60,2 \%$ entre atestado original e atestado refeito, sendo que as anomalias congênitas perfizeram o maior percentual de concordância $(92,0 \%)$. Na distribuição por grupo de causa do atestado original, o percentual mais elevado foi o das doenças infecciosas e parasitárias, com $21,6 \%$ do total, com a gastroenterite representando $43,2 \%$ deste grupo.

UNITERMOS: Mortalidade. Atestado de óbito. Morte, causa básica.

\section{1 - INTRODUÇÃO}

A importância do atestado de óbito para fornecer informações sobre a saúde de uma população foi reconhecida há séculos, tendo este documento de estatística vital se constituído na base da moderna epidemiologia ${ }^{3}$.

Vários estudos publicados, referentes à qualidade das informações contidas no atestado de óbito, têm demonstrado que, embora seja adotado um modelo uniforme de atestado de óbito e uma definição precisa da causa básica, as estatísticas de mortalidade não são, ainda, inteiramente corretas. Tem sido invocado, frequientemente, como justificativa, o fato de nem sempre, nos currículos das escolas médicas, ser dada a devida atenção à importância do correto preenchimento da causa de óbito.

Não raro ocorre que o médico se depara, pela primeira vez, com um atestado de óbito, no momento em que se vê na contingência real de preenchê-lo. Passa, então, a ver este documento somente como exigência legal, com vistas à finalidade de sepultamento e poucas vezes compreende a importância que as informações neles registradas têm para a saúde pública ${ }^{2}$.

O desconhecimento, por grande parte dos médicos, sobre a maneira correta de preencher o atestado, faz com que eles assinalem, muitas vezes, a causa básica de maneira incorreta, de tal forma

- Academia da 11. fase do Curso de Graduação em Medicina da Universidade Federal de Santa Catarina, $2 \%$ semestre de 1983.

* Da Unidade de Documentação e Informática da Saúde, da Secretaria da Saúde do Estado de Santa Catarina - Av. Osmar Cunha, 15 - 88000 - Florianópolis, SC, Brasil.

** Do Departamento de Saúde Pública do Centro de Clências da Saúde da Universidade Federal de Santa Catarina - Rua Ferreira Lima, 26 - 88000 - Florianópolis, SC - Brasil.

*** Do Departamento de Pediatria do Centro de Ciências da. Saúde da Universidade Federal de Santa Catarina - 88000 - Florianópolis, SC - Brasil. 
STROZZI, G.M. et al. Estudo de causa básica de óbitos de menores de 15 anos, ocorridos em hospital de Florianópolis, SC (Brasil), em 1982. Rev. Saúde públ., S. Paulo, 19:123-32, 1985.

que, mesmo aplicando as regras de seleção, ela não será codificada ${ }^{1}$.

O diagnóstico de saúde de uma comunidade é comumente levantado pelo uso de indicadores de saúde os quais, pela sua própria definição, indicam níveis acima ou abaixo de um padrão pré-estabelecido. Porém, para a implantação de um programa de saúde capaz de contribuir para transformar a realidade expressa pelos indicadores de saúde, precisam ser apuradas informações sobre os determinantes do diagnóstico encontrado. A mortalidade proporcional por causa ou grupos de causa de morte constitui uma das melhores fontes de informação sobre mortalidade e, muito embora passível de erro, ainda é a mais válida à disposição do investigador.

A validade das estatísticas de mortalidade é avaliada em estudos específicos, para que o reconhecimento da sua importância e a segurança do seu uso sejam cada vez maiores.

$O$ estudo de mortalidade tem importância variada, dependendo do grau de detalhamento em que é abordado, seja por indicadores ou pela preocupação em aumentar e manter a validade das informações.

Em estudos de mortalidade, quando se trabalha exclusivamente com atestados de óbito, é necessário que se considere as limitações do documento, tendo em vista que, em nosso meio, as informações nele contidas ainda são deficientes e de fidedignidade relativa, sendo de fundamental importância o retorno à fonte primária, o prountária médico, para complementação e correção de dados.

Objetiva-se, com o presente estudo, conhecer a distribuição das causas de mortalidade de menores de 15 anos, dos óbitos ocorridos em hospital infantil e identificar possíveis sub-registros de óbitos.

\section{2 - METODOLOGIA}

Considerou-se como população de estudo o total da clientela de um hospital e que tivesse falecido no período de $1 .^{\circ}$ de janeiro a 31 de dezembro de 1982 . O hospital escolhido para o presente estudo é uma unidade da Fundação Hospitalar de Santa Catarina, destinada a receber pacientes menores de 15 anos, de todas as categorias sociais. Possui um total de 212 leitos. E um hospital-escola que mantém um serviço de residência médica em pediatria clínica e cirúrgica e é campo de estágio dos diversos cursos de graduação do Centro de Ciências da Saúde, da Universidade Federal de Santa Catarina. E o único hospital infantil de Florianópolis.

No livro de registro de ocorrências do Hospital foram observadas referências a 190 óbitos no período considerado. No Serviço de Arquivo Médico e Estatística (SAME), do referido hospital foram encontrados prontuários e respectivos atestados de 161 óbitos dos 190 óbitos registrados, como também atestados de 19 óbitos de pacientes externos, ocorridos no Setor de Emergência, para os quais não foram abertos prontuários; não foram encontrados nem prontuários nem atestados dos 10 óbitos restantes. Confrontando as informações obtidas no hospital com as existentes na Unidade de Documentação e Informática de Saúde, da Secretaria da Saúde do Estado de Santa Catarina (SS), observou-se que os 161 óbitos, para os quais o hospital abriu prontuário e tinha arquivadas as cópias do atestado de óbito, estavam registrados na SS; os 10 óbitos registrados no livro de ocorrências do hospital e cujos prontuários e atestados não constavam do arquivo do SAME, também estavam registrados na SS com cópia disponível dos atestados de óbito; observou-se, ain$\mathrm{da}$, que a SS não dispunha de registros ou cópias dos atestados dos 19 óbitos restantes, o que, provavelmente, indica um sub-registro de informações entre 
STROZZI, G.M. et al. Estudo de causa básica de óbitos de menores de 15 anos, ocorridos em hospital de Florianópolis, SC (Brasil), em 1982. Rev. Saúde públ., S. Paulo, 19:123-32, 1985.

Hospital, SS e Cartório de Registro Civil.

As informações relacionadas aos atestados de óbito originais foram trabalhadas a partir dos 171 óbitos oficialmente registrados na SS. De posse das cópias dos 171 atestados de óbitos, as informações foram classificadas por grupo de causa de morte e faixa etária e a distribuição por grupo comparada à de Florianópolis e Santa Catarina.

Para refazer os atestados de óbito foram utilizados os 161 óbitos que contavam com a história clínica registrada nos prountários arquivados no SAME do hospital. Refeitos os atestados, estes foram submetidos às regras de codificação contidas no Manual ${ }^{4}$ e posteriormente comparados aos atestados originais, com vistas a verificar possíveis sub-registros de causa de óbito.

Os dados obtidos dos atestados de óbito e dos prountários foram tabulados manualmente.

\section{3 - RESUltados e COMENTÁRIOS \\ 3.1 - Mortalidade proporcional por grupos de causa}

Utilizando as informações contidas nos atestados de óbito, oficialmente registrados na SS como de ocorrência no Hospital, foram estudadas as distribuições das causas básicas dos óbitos, apresentados por grupo de causas e estes comparados com os percentuais distribuídos para o município de Florianópolis e o Estado de Santa Catarina (Tabela 1).

\section{TABELA 1}

Óbitos de menores de 15 anos registrados na SS como de ocorrência no Hospital (1982), em comparação com o total de óbitos do mesmo grupo etário, ocorridos no município de Florianópolis e no Estado de Santa Catarina no ano de 1980.

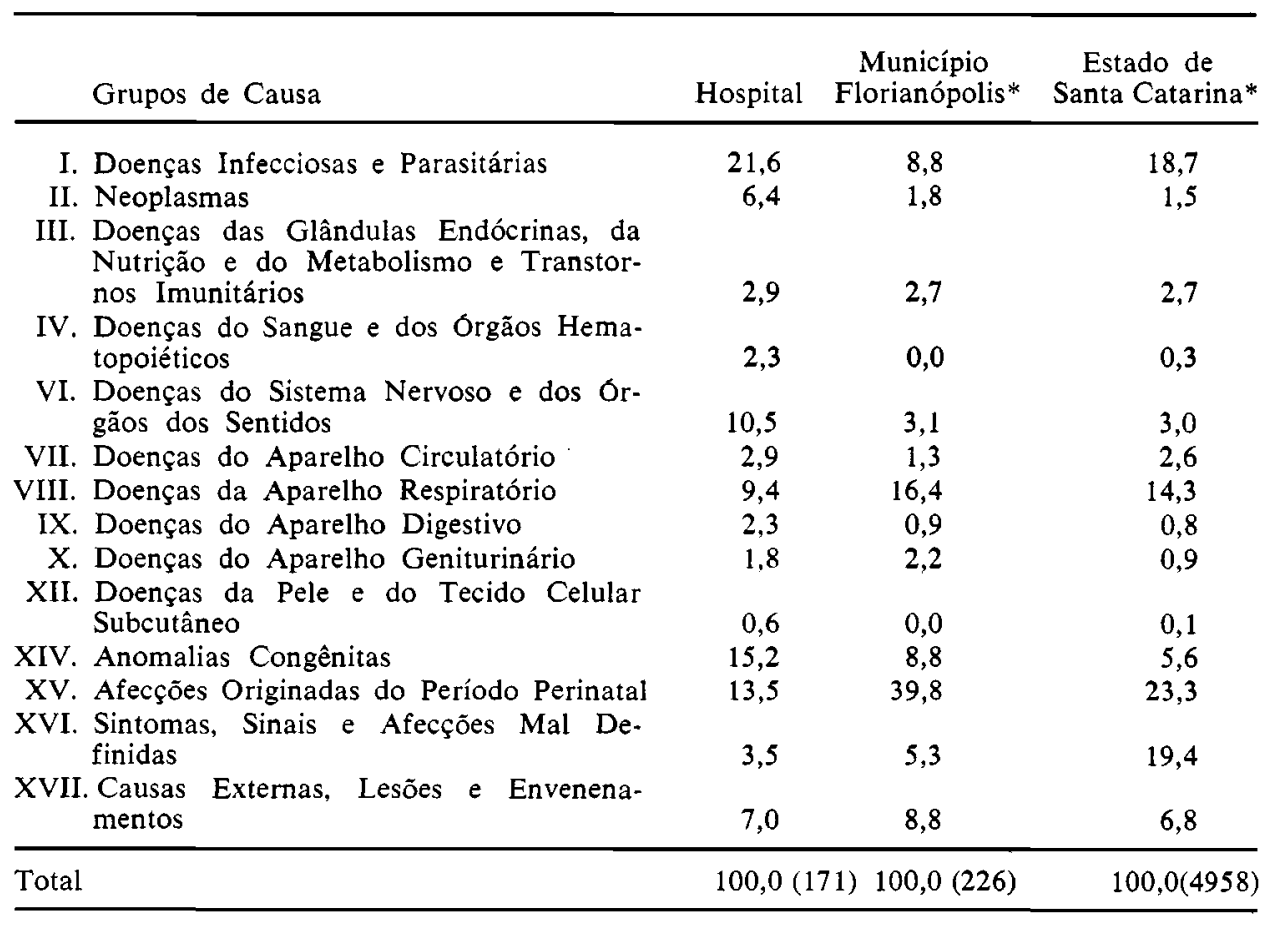

* Unidade de Documentação e Informática da Secretaria Estadual de Saúde - Santa Catarina. 
STROZZI, G.M. et al. Estudo de causa básica de óbitos de menores de 15 anos, ocorridos em hospital de Florianópolis, SC (Brasil), em 1982. Rev. Saúde públ., S. Paulo, 19:123-32, 1985.

Comparando os valores percentuais por grupo de causa atribuído ao Hospital com os do município de Florianópolis e o Estado de Santa Catarina, verifica-se que a distribuição de freqüência entre os grupos tem elevada variação. Os grupos de causa mais freqüentes entre os óbitos do Hospital foram as Doenças Infecciosas e Parasitátias $(21,6 \%)$, seguidas das Anomalias Congênitas $(15,2 \%)$ e das Afecções Originadas no Período Perinatal (13,5\%); os de Florianópolis foram Afecções Originadas no Período Perinatal $(39,8 \%)$ e Doenças do Aparelho Respiratório (16,4\%); e os de Santa Catarina foram Afecções Originadas no Período Perinatal $(23,3 \%)$, Sintomas, Sinais e Afecções Mal Definidas $(19,4 \%)$ e Doenças Infecciosas e Parasitárias $(18,7 \%)$.

A percentagem de Afecções Originadas no Período Perinatal identificadas no Hospital, quando comparada às atribuídas ao município de Florianópolis e o Estado de Santa Catarina, foi mais bai$\mathrm{xa}$, provavelmente porque $\mathrm{o}$ grupo de causa de óbito tem como local de ocorrência preferencial as Maternidades.

As proporções de morte por grupo de causa do Hospital, do município de Florianópolis e do Estado de Santa Catarina foram correlacionadas entre si, utilizando-se o coeficiente de correlação (r), testado em pares. A correlação entre o Hospital versus Florianópolis foi de 0,54; Hospital versus Santa Catarina foi de 0,56; enquanto que Florianópolis versus Santa Catarina foi de 0,77 . Isto indica que há uma correlação mais fraca entre Hospital e Florianópolis e entre Hospital e Santa Catarina, enquanto que entre estes dois últimos a correlação é mais forte. Percebe-se, deste modo, apesar das limitações deste teste, que há nítida diferença entre a proporção de mortes por grupo de causas do Hospital em relação ao Estado de Santa Catarina e ao município de Florianópolis, que são semelhantes entre si.

\section{2 - Comparação entre a causa básica se- lecionada no atestado de óbito original e no atestado de óbito refeito}

Após identificados e classificados por grupos de causa, as informações dos atestados de óbito originais foram comparados com a dos atestados refeitos, com vistas à verificação da concordância das causas básicas. No conjunto, observou-se um percentual de concordância de $60,2 \%$ entre a causa básica informada no atestado de óbito original e atribuída no atestado refeito, ou seja, de cada 5 atestados originais, 2 foram refeitos com causa básica diferente da original. Embora este fato não altere a mortalidade quantitativamente, altera-a qualitativamente, o que ressalta a importância dessa abordagem metodológica.

A partir dos dados da Tabela 2 pode-se verificar casos de sub e superestimação qualitativa que podem ser corrigidos quando empregada a metodologia do presente trabalho.

Com relação à subestimação exemplifica-se o que foi observado nos grupos de causas de Doenças Infecciosas e Parasitárias e Anomalias Congênitas. Nestes grupos foi verificado um total de 34 casos de óbito por Doenças Infecciosas e Parasitárias quando, na opinião dos pesquisadores, o total deveria ser de 45 e, ainda, da totalidade dos casos, originalmente definidos, somente 23 foram coincidentes; os atestados de óbito originais apontaram um total de 25 óbitos por Anomalias Congênitas, enquanto que os pesquisadores definiram $31 \mathrm{e}$, ainda, da totalidade dos casos, originalmente definidos, somente 23 foram coincidentes. Neste exemplo, verificou-se uma subestimação de $24,4 \%$ para as Doenças Infecciosas e Parasitárias e de $13,6 \%$ para as Anomalias Congênitas.

A superestimação pode ser exemplificada pelo que foi verificado no grupo de Doenças do Aparelho Respiratório, quando os atestados de óbito originais definiam 14 óbitos por esta causa e os 
STROZZI, G.M. et al. Estudo de causa básica de óbitos de menores de 15 anos, ocorridos em hospital de Florianópolis, SC (Brasil), em 1982. Rev. Saúde públ., S. Paulo, 19:123-32, 1985.

\section{TABELA 2}

Distribuição dos óbitos por grupos de causa em relação à frequiência nos atestados originais e refeitos (dos mesmos casos).

\begin{tabular}{|c|c|c|c|c|c|c|}
\hline \multirow{4}{*}{ Grupo de Doenças } & \multicolumn{6}{|c|}{ Atestado de 6́bito } \\
\hline & \multirow{3}{*}{$\begin{array}{l}\text { Ne } \\
\text { Original } \\
\quad(1)\end{array}$} & \multirow{3}{*}{$\begin{array}{l}\text { No } \\
\text { Refeito } \\
\text { (2) }\end{array}$} & 1 & & $\mathbf{X}$ & 2 \\
\hline & & & \multicolumn{2}{|c|}{ Sim } & \multicolumn{2}{|c|}{ Não } \\
\hline & & & No & $\%$ & No & $\%$ \\
\hline I. Doenças Infecciosas e Parasitárias & 34 & 45 & 23 & 67,6 & 11 & 32,4 \\
\hline II. Neoplasmas & 11 & 12 & 10 & 90,9 & 1 & 9,1 \\
\hline $\begin{array}{l}\text { III. Doenças das Glândulas Endócri- } \\
\text { nas, da Nutrição e do Metabo- } \\
\text { lismo e Transtornos Imunitários }\end{array}$ & 5 & 2 & 2 & 40,0 & 3 & 60,0 \\
\hline $\begin{array}{l}\text { IV. Doenças do Sangue e dos Orgãos } \\
\text { Hematopoiéticos }\end{array}$ & 4 & 1 & 1 & 25,0 & 3 & 75,0 \\
\hline $\begin{array}{l}\text { VI. Doenças do Sistema Nervoso e } \\
\text { dos Orgãos dos Sentidos }\end{array}$ & 18 & 22 & 13 & 72,2 & 5 & 27,8 \\
\hline VII. Doenças do Aparelho Circulatório & 5 & 4 & 2 & 40,0 & 3 & 60,0 \\
\hline VIII. Doenças do Aparelho Respiratório & 14 & 8 & 4 & 25,0 & 10 & 75,0 \\
\hline IX. Doenças do Aparelho Digestivo & 4 & 6 & 2 & 50,0 & 2 & 50,0 \\
\hline X. Doenças do Apar. Geniturinário & 3 & 1 & 0 & 00,0 & 3 & 100,0 \\
\hline XIV. Anomalias Congênitas & 25 & 31 & 23 & 92,0 & 2 & 8,0 \\
\hline $\begin{array}{l}\text { XV. Afecções Originadas no Período } \\
\text { Perinatal }\end{array}$ & 23 & 19 & 10 & 43,5 & 13 & 56,5 \\
\hline $\begin{array}{l}\text { XVI. Sintomas, Sinais e Afecções Mal } \\
\text { Definidas }\end{array}$ & 6 & 1 & 0 & 00,0 & 6 & 100,0 \\
\hline $\begin{array}{l}\text { XVII. Causas Externas, Lesões e En- } \\
\text { venenamentos }\end{array}$ & 9 & 9 & 7 & 77,8 & 2 & 22,2 \\
\hline Total & 161 & 161 & 97 & 60,2 & 64 & 39,8 \\
\hline
\end{tabular}

pesquisadores somente 8 casos, com uma concordância de 4 casos e uma superstimação de 75,0\% em grande parte devida à Broncopneumonia.

Os dados da Tabela 3 mostram que, se considerarmos os atestados de óbito originais, teremos uma distribuição de frequiência proporcional diferente daquela dos atestados refeitos. As diferenças entre os percentuais originais e refeitos variam desde $-83,8$ até $+48,0$ pontos percentuais, sendo o grupo XVII (Causas Externas, Lesões e Envenenamentos) o único que teve sua proporção mantida.

\section{3 - Distribuição da mortandade por gru- po de causa, idade e sexo}

Os dados da Tabela 4 permitem verificar que $65,5 \%$ dos 171 óbitos ocorreram em menores de um ano de idade; destes, $41,5 \%$ tinham idades compreendidas entre 28 dias e um ano. Das demais faixas etárias consideradas, a que perfez maior percentual foi a de 2 a 7 anos, com $17,5 \%$, enquanto que a faixa etária de 12 a 14 anos obteve o menor percentual, ou seja, $1,8 \%$.

Estas informações complementam-se com os dados da Tabela 5, onde se 
STROZZI, G.M. et al. Estudo de causa básica de óbitos de menores de 15 anos, ocorridos em hospital de Florianópolis, SC (Brasil), em 1982. Rev. Saúde públ., S. Paulo, 19:123-32, 1985.

\section{TABELA 3}

Mortalidade proporcional por grupo de causa dos atestados originais, refeitos e a diferença percentual encontrada.

\begin{tabular}{|c|c|c|c|}
\hline Grupo de Causa & $\begin{array}{l}\text { Original } \\
(\%)\end{array}$ & $\begin{array}{l}\text { Atestado de } \\
\text { Refeito } \\
(\%)\end{array}$ & $\begin{array}{c}\text { Fig } \\
\text { (Pontos } \\
\text { percentuais) } \\
1 \times 2 \\
\end{array}$ \\
\hline I Doenças Infecciosas e Parasitárias & $21,1(34)$ & $28,0(45)$ & $+32,7$ \\
\hline II. Neoplasmas & $6,8(11)$ & $7,5(12)$ & $+8,8$ \\
\hline $\begin{array}{l}\text { III. Doenças das Glândulas Endócrinas, da } \\
\text { Nutrição e do Metabolismo e Trans- } \\
\text { tornos Imunitários }\end{array}$ & $3,1(5)$ & $1,2(2)$ & $-61,3$ \\
\hline $\begin{array}{l}\text { IV. Doenças do Sangue e dos Orgãos } \\
\text { Hematopoiéticos }\end{array}$ & $2,5(4)$ & $0,6(1)$ & $-76,0$ \\
\hline $\begin{array}{l}\text { VI. Doenças do Sistema Nervoso e dos } \\
\text { Orgãos dos Sentidos }\end{array}$ & $11,2(18)$ & $13,7(22)$ & $+22,3$ \\
\hline VII. Doenças do Aparelho Circulatório & $3,1(5)$ & $2,5(4)$ & $-19,4$ \\
\hline VIII. Doenças do Aparelho Respiratório & $8,7(14)$ & $5,0(8)$ & $-42,5$ \\
\hline IX. Doenças do Aparelho Digestivo & $2,5(4)$ & $3,7(6)$ & $+48,0$ \\
\hline X. Doenças do Aparelho Geniturinário & $1,9(3)$ & $0,6(1)$ & $-68,4$ \\
\hline XIV. Anomalias Congênitas & $15,5(25)$ & $19,3(31)$ & $+24,5$ \\
\hline $\begin{array}{l}\text { XV. Afecções Originadas no Período Pe- } \\
\text { rinatal }\end{array}$ & $14,3(23)$ & $11,8(19)$ & $-17,5$ \\
\hline $\begin{array}{l}\text { XVI. Sintomas, Sinais e Afecções Mal De- } \\
\text { finidas }\end{array}$ & $3,7(6)$ & $0,6(1)$ & $-83,8$ \\
\hline $\begin{array}{l}\text { XVII. Causas Externas, Lesões e Envene- } \\
\text { namentos }\end{array}$ & $5,6(9)$ & $5,6(9)$ & 00,0 \\
\hline Total & $100,0(161)$ & $100,0(161)$ & \\
\hline
\end{tabular}

OBS.: Os valores entre parêntesis referem-se ao número de casos.

TABELA 4

Obitos por grupos etários - atestado de óbito original.

\begin{tabular}{|c|c|c|c|}
\hline Grupos Etários & $\begin{array}{c}\text { Número } \\
\text { de } \\
\text { Obitos }\end{array}$ & $\%$ & $\%$ Acumulada \\
\hline$<7 \mathrm{~d}$ & 22 & 12,9 & 12,9 \\
\hline $7 \mathrm{~d}-28 \mathrm{~d}$ & 19 & 11,1 & 24,0 \\
\hline $28 \mathrm{~d} \longmapsto$ 1a & 71 & 41,5 & 65,5 \\
\hline $1 a-2 a$ & 12 & 7,0 & 72,5 \\
\hline $2 a-7 a$ & 30 & 17,5 & 90,0 \\
\hline $7 a-10 a$ & 10 & 5,9 & 95,9 \\
\hline $10 \mathrm{a}-12 \mathrm{a}$ & 4 & 2,3 & 98,2 \\
\hline $12 \mathrm{a}-14 \mathrm{a}$ & 3 & 1,8 & 100,0 \\
\hline Total & 171 & 100,0 & \\
\hline
\end{tabular}




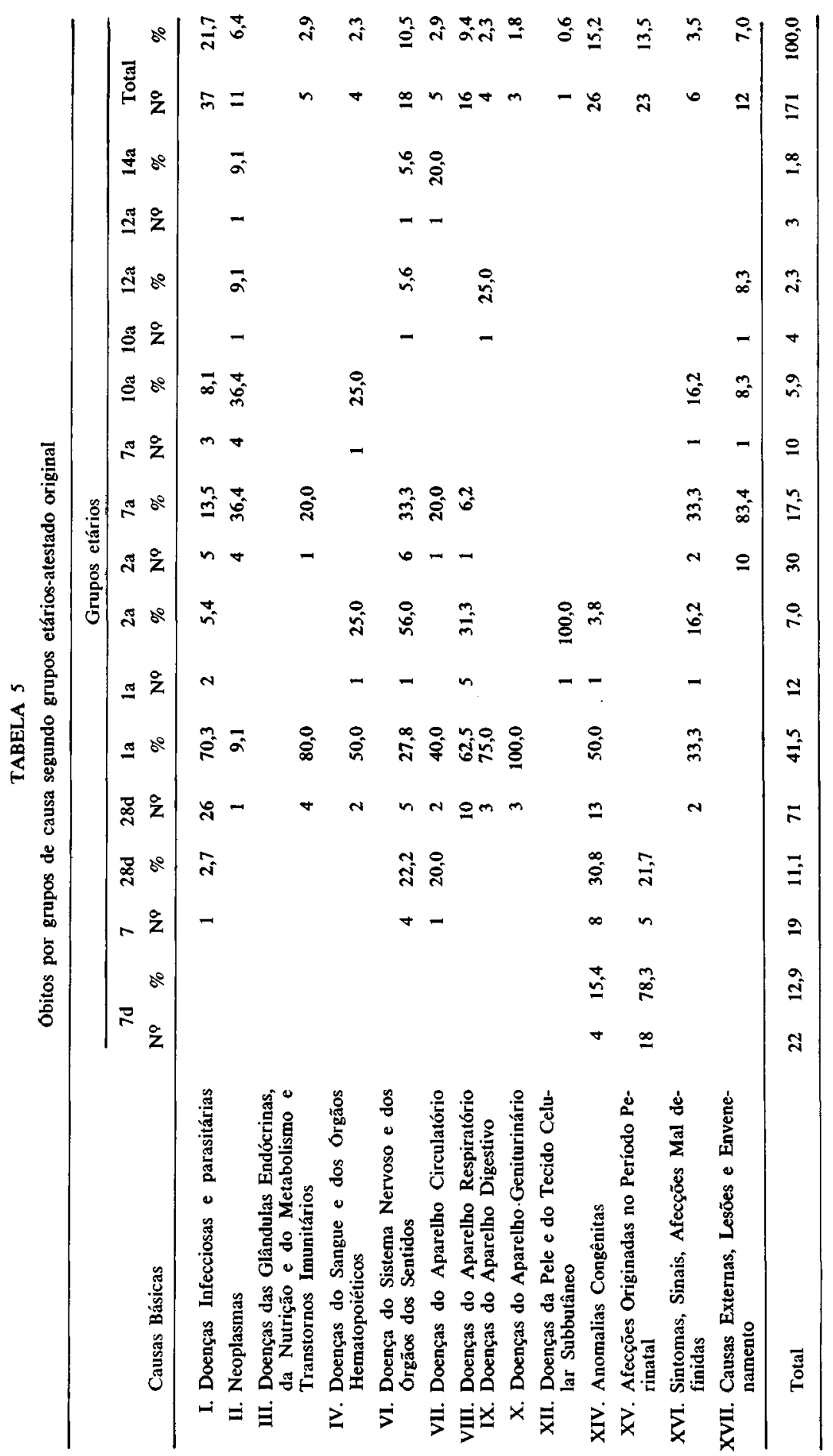


STROZZI, G.M. et al. Estudo de causa básica de óbitos de menores de 15 anos, ocorridos em hospital de Florianópolis, SC (Brasil), em 1982. Rev. Saúde públ., S. Paulo, 19:123-32, 1985.

observa a distribuição dos grupos de causa de óbito por grupos etários.

Entre os menores de um ano os grupos de causas mais frequientes foram Doenças Infecciosas e Parasitárias, Anomalias Congênitas e Afecções Originadas no Período Perinatal.

As Doenças do Aparelho Respiratório foram as causas mais freqüentes dos óbitos de crianças de um a 2 anos, enquanto que no grupo de 2 a 7 anos foram as Causas Externas, Lesões e Envenenamentos. Embora o grupo dos Sintomas, Sinais e Afecções Mal Definidas representem somente $3,5 \%$ dos 171 óbitos, mostram, na prática, a impropriedade qualitativa dos atestados de óbito e também a falta de encaminhamento destes casos para o Instituto Médico Legal para conclusão diagnóstica, o que reflete perda qualitativa de informações.

A mortalidade segundo o sexo (Tabela 6) mostrou, no global, pequena predominância do sexo masculino $(52,1 \%)$ sobre o sexo feminino $(47,9 \%)$.

$\mathrm{Na}$ faixa etária estudada, os óbitos por Causas Externas, Lesões e Envenenamentos foram mais frequientes no se-

TABELA 6

Obitos por grupo de causas segundo sexo-atestado original

\begin{tabular}{|c|c|c|c|c|c|c|}
\hline \multirow[b]{3}{*}{ Grupo de causa } & \multicolumn{4}{|c|}{ Sexo } & & \\
\hline & \multicolumn{2}{|c|}{ Masc. } & \multicolumn{2}{|c|}{ Fem. } & \multicolumn{2}{|c|}{ Total } \\
\hline & No & $\%$ & No & $\%$ & NQ & $\%$ \\
\hline I. Doenças Infecciosas e Parasitárias & 17 & 45,9 & 20 & 54,1 & 37 & 21,6 \\
\hline II. Neoplasmas & 8 & 72,7 & 3 & 27,2 & 11 & 6,4 \\
\hline $\begin{array}{l}\text { III. Doenças das Glândulas Endócrinas } \\
\text { de Nutrição e do Metabolismo e } \\
\text { Transtornos Imunitários }\end{array}$ & 3 & 60,0 & 2 & 40,0 & 5 & 2,9 \\
\hline $\begin{array}{l}\text { IV. Doenças do Sangue e dos órgãos } \\
\text { Hematopoiéticos }\end{array}$ & 1 & 25,0 & 3 & 75,0 & 4 & 2,3 \\
\hline $\begin{array}{l}\text { VI. Doenças do Sistema Nervoso e dos } \\
\text { Orgãos dos Sentidos }\end{array}$ & 8 & 44,4 & 10 & 55,6 & 18 & 10,5 \\
\hline VII. Doenças do Aparelho Circulatório & 4 & 80,0 & 1 & 20,0 & 5 & 2,9 \\
\hline VIII. Doenças do Aparelho Respiratório & 10 & 62,5 & 6 & 37,5 & 16 & 9,4 \\
\hline IX. Doenças do Aparelho Digestivo & 2 & 50,0 & 2 & 50,0 & 4 & 2,3 \\
\hline X. Doenças do Aparelho Geniturinário & 1 & 33,3 & 2 & 66,7 & 3 & 1,8 \\
\hline $\begin{array}{l}\text { XII. Doenças da Pele e do Tecido Ce- } \\
\text { lular Subcutâneo }\end{array}$ & 0 & 0,0 & 1 & 100,0 & 1 & 0,6 \\
\hline XIV. Anomalias Congênitas & 12 & 46,2 & 14 & 53,8 & 26 & 15,2 \\
\hline $\begin{array}{l}\text { XV. Afecções Originadas no Período } \\
\text { Perinatal }\end{array}$ & 14 & 60,9 & 9 & 39,1 & 23 & 13,5 \\
\hline $\begin{array}{l}\text { XVI. Sintomas, Sinais e Afecções Mal } \\
\text { Definidas }\end{array}$ & 4 & 66,7 & 2 & 33,3 & 6 & 3,5 \\
\hline $\begin{array}{l}\text { XVII. Causas Externas, Lesões e Envene- } \\
\text { namentos }\end{array}$ & 5 & 41,7 & 7 & 58,3 & 12 & 7,0 \\
\hline Total & 89 & 52,1 & 82 & 47,9 & 171 & 100,0 \\
\hline
\end{tabular}


STROZZI, G.M. et al. Estudo de causa básica de óbitos de menores de 15 anos, ocorridos em hospital de Florianópolis, SC (Brasil), em 1982. Rev. Saúde públ., S. Paulo, 19:123-32, 1985.

xo feminino, o que não coincide com os resultados de Mello Jorge e Marques ${ }^{5}$, provavelmente face à composição populacional diferente. Quanto aos óbitos por Afecções Originadas no Período Perinatal, o sexo masculino contribuiu com $60,9 \%$ dos óbitos deste grupo. Incidência maior ainda foi verificada no grupo dos neoplasmas com um percentual de $72,7 \%$ de masculinos.

\section{4 - CONCLUSÓES}

A distribuição da mortalidade por causa básica, no Hospital difere das distribuições análogas do Município de Florianópolis e do Estado de Santa Catarina.

A concordância entre os atestados de óbito originais e refeitos difere entre os grupos de causa e apresenta um global de $60,2 \%$.

A mortalidade proporcional entre os atestados de óbitos originais e atestados refeitos são diferentes entre si, indicando que existe uma probabilidade de erro inaparente no estudo de mortalidade proporcional.

Dentre as maiores percentagens por grupos de causa de inorte, foram encontradas as Doenças Infecciosas e Parasitárias: $21,6 \%$ nos atestados originais, aumentando para $28,0 \%$ nos refeitos; seguidas das Anomalias Congênitas; $15,5 \%$ e $19,3 \%$, e Afecções Originadas no Período Perinatal: $14,3 \%$ e $11,8 \%$, respectivamente.

As Gastroenterites apareceram como a maior causa isolada de morte $(9,4 \%$ da totalidade dos óbitos), o que faz supor a associação com desnutrição e que as crianças quando chegam ao hospital já se encontram em estado irreversível de infecção/desidratação;

As causas externas representaram $33,3 \%$ dos óbitos do grupo etário de 2 a 7 anos, principalmente devido a atropelamentos, o que evidencia a necessidade de educação para o trânsito já a nível pré-escolar, tentando assim diminuir a mortalidade por esta causa.

STROZZI, G.M. et al. [A study of the basic cause of death in children under 15, during 1982, in Children Hospital (Florianópolis, Santa Catarina, Brasil)\}. Rev. Saúde públ., S. Paulo, 19:123-32, 1985.

ABSTRACT: Death certificates were analysed according to the basic cause of death. Out of the total number of deaths which had occured in Children Hospital (Florianópolis, SC, Brazil), 161 were considered valid for the purpose of this study. With the information obtained from the respective hospital file, the death certificates were filled out again, and then compared to the original ones. Agreement was observed in $60.2 \%$ of cases between the original death certificates and the re-filled ones, and Congenital Malformations gave the highest percentage of agreement (92.0). When frequency distribution by cause of death was studied in the original death certificates, the highest percentage appeared to be Infectious and Parasitic Diseases (21.0) and of those Enteritis accounted for $43.2 \%$.

UNITERMS: Mortality. Death certificates. Death, causes. 
STROZZI, G.M. et al. Estudo de causa básica de óbitos de menores de 15 anos, ocorridos em hospital de Florianópolis, SC (Brasil), em 1982. Rev. Saúde públ., S. Paulo, 19:123-32, 1985.

\section{REFERENCIAS BIBLIOGRÁFICAS}

1. LAURENTI, R. A análise da mortalidade por causa básica e por causas múltiplas. Rev. Saúde públ., S. Paulo, 8: 421-35, 1974.

2. LAURENTI, $R$. \& MELLO JORGE, M.H.P. de. $O$ atestado de óbito. São Paulo, Centro da OMS para Classificação de Doenças em Português, 1981.

3. MAC MAHON, B. \& PUGH, T.F. Epidemiology: principles and methods. Boston, Little Brown, 1970.

4. MANUAL da Classificação Estatística Internacional de Doenças, Lesões e Cau- sas de Obitos; 9. a Revisão. São Paulo, Centro da OMS para Classificação de Doenças em Português, 1980.

5. MELLO JORGE, M.H.P. de \& MARQUES, M.B. Acidentes na infância no Brasil. [Trabalho apresentado na Reunião de Avaliação das Investigações Nacionais sobre Acidentes na Infância, Ankara, Turquia, 1982]

Recebido para publicação em 14/04/1984

Reapresentado em 23/01/1985

Aprovado para publicação em 30/01/1985 